\title{
Role of Microendodontics in Detection of Root Canal Orifices: A Comparative Study between Naked Eye, Loupes and Surgical Operating Microscope
}

\author{
Authors \\ Dr Neelam Mittal, Dr Suraj Arora \\ Department of Conservative Dentistry \& Endodontics \\ JCD Dental College, Barnala Road, Sirsa, Haryana 1250555 India \\ Email: surajarorasgrd@yahoo.co.in Mobile Number: 9041828632
}

\begin{abstract}
Aim: The aim of this study was to compare the detection rate of root canal orifices by three different methods: naked eye, with surgical loupes and under a surgical operating microscope.

Material and methods: Sixty extracted permanent maxillary molars and sixty extracted permanent mandibular molars were collected. Access cavity was prepared. After an access cavity, the long shank spoon excavator was used to remove the contents of the chamber and irrigation was done with $5 \%$ sodium hypochlorite. The existence of each orifice was recorded when a K-file \#8 or \#10 pushed into the orifice was able to stand by itself.

The teeth were divided into four groups (1-4). In each group the number of root canal orifices were recorded using naked eye (Gp 1), loupes (Gp 2), surgical operating microscope (Gp 3) and after tooth clearing technique (Gp 4). Tooth clearing technique gave the actual number of root canal orifices and was used as a standard for comparison.

Statistical analysis: Chi square test was used.

Result: There was significant difference between groups 1 and 3; and groups 2 and 3. Although the number of orifices detected was greater with loupes $(G p 2)$ than eye $(G p 1)$ but it was not significant.

Conclusion: The number of orifices detected increased with increasing magnification. Experience of the operator influences the detection rate of root canal orifices. The microscope could more accurately detect orifices statistically than the other two methods.
\end{abstract}

Keywords: loupes, microscope, molars, orifice

\section{Introduction}

Natural teeth function more efficiently than artificial replacement and thus preservation of the natural dentition is the main goal of dental treatment. The main objective of endodontic therapy is the thorough mechanical and chemical cleansing of the entire pulp cavity and its complete obturation with an inert filling material. Failures in treatment occur despite rigid adherence to this basic principle. The main reasons for this 
failure are incomplete canal obturation and an untreated canal. A canal is often left untreated because the dentist fails to recognize its presence. A dentist must have a thorough knowledge of root canal morphology before he can successfully treat a tooth endodontically.

Maxillary and mandibular molars can have quite variations as to the number of root canals. Hess ${ }^{1}$ (1925) reported the prevelance of three root canals in mandibular permanent molar teeth to $78 \%$. Skidmore and Bjornal $^{2}$ (1971) demonstrated the prevelance of two root canals in the distal root of permanent mandibular teeth to almost $30 \%$.

The root canal system of the mesiobuccal root of maxillary molars has been extensively investigated in both ex vivo and in vivo studies. Ex vivo studies indicate that a second root canal is present in $55-69 \%$ of these roots (Pineda and kuttler $^{3}$ 1972, Pineda ${ }^{4} 1973$, Seidberg ${ }^{5}$ et al 1973 , Pomeranz and Fishelberg ${ }^{6}$ 1974, Vertucci $^{7}$ 1974).

The present study was designed with the purpose to compare the detection rate of root canal orifices by three different methods : naked eye, surgical loupes and under an operating microscope.

\section{Materials and methods}

Sixty extracted permanent maxillary molars (30 maxillary first and 30 maxillary second) and sixty extracted permanent mandibular molars (30 mandibular first and 30 mandibular second) were collected from the dental OPD, Faculty of Dental Sciences, Institute of Medical Sciences, B.H.U, Varanasi. The teeth were thoroughly washed under tap water to remove the saliva and blood. The root surfaces were cleaned of the soft ad hard deposits using hand scaling instruments and scrubbing under tap water and stored in distilled water. A rhomboidal and trapezoidal access cavity was made in maxillary and mandibular molars respectively. After an access cavity, the long shank spoon excavator was used to remove the contents of the chamber and irrigation was done with 5\% sodium hypochlorite. DG-16 endodontic explorer was used to explore the pulp chamber floor. Dentine overhangs were removed with swan necked LN bur to open subpulpal groove to locate extra canal orifices.

Exploration of the groove connecting the canal orifice was done using K-files \#6, \#8, \#10. The existence of each orifice was recorded when a Kfile \#8 or \#10 pushed into the orifice was able to stand by itself.

Maxillary and mandibular molars were divided into four goups:

\section{Group-1}

After access cavity, the number of canal orifices in each root were recorded by naked eye.

\section{Group 2}

All the teeth were again observed under surgical loupes $(2.3 \mathrm{x})$ by Hien. If dentine overhang was present, then it was removed using $\mathrm{LN}$ bur to find another canal orifice and the number of canal orifices in each root were recorded.

\section{Group 3}

The teeth were observed through surgical operating microscope of $3.4 \mathrm{x}$ to $21 \mathrm{x}$ magnification at $200 \mathrm{~mm}$ distance from the tooth in direct vision. The number of root canal orifices in each root were recorded (Fig 1).

\section{Group 4}

The tooth clearing technique was used to detect the actual number of root canal orifices as following

India ink was injected into the pulp chamber and aspirated from the apical foramen. The teeth were placed in 5\% nitric acid solution for 72 hours and the solution was changed every 24 hours. The teeth were rinsed in running tap water for 4 hours and placed in $80 \%$ alcohol for 24 hours, followed by 24 hours in $90 \%$ alcohol and finally in $100 \%$ absolute ethyl alcohol for 24 hours to dehydrate the specimens. The teeth were then cleared in methyl salicylate solution for overnight. The actual number of root canal orifices were then recorded under surgical operating microcope (Fig 2). 


\section{JMSCR Vol||3||Issue||10||Page 7810-7816||October 2015}
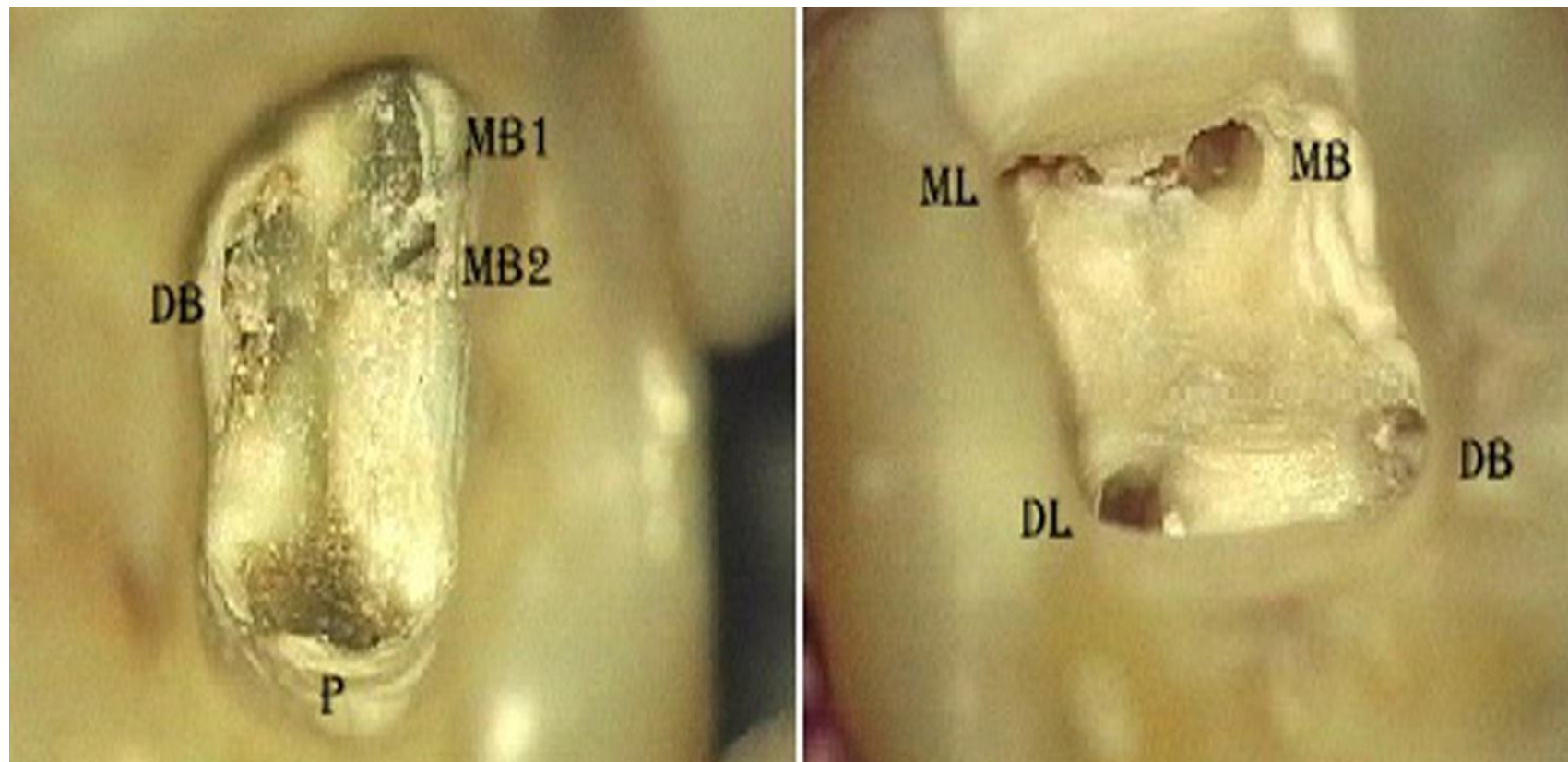

Fig. 1: Maxillary and mandibular molars under operating microscope
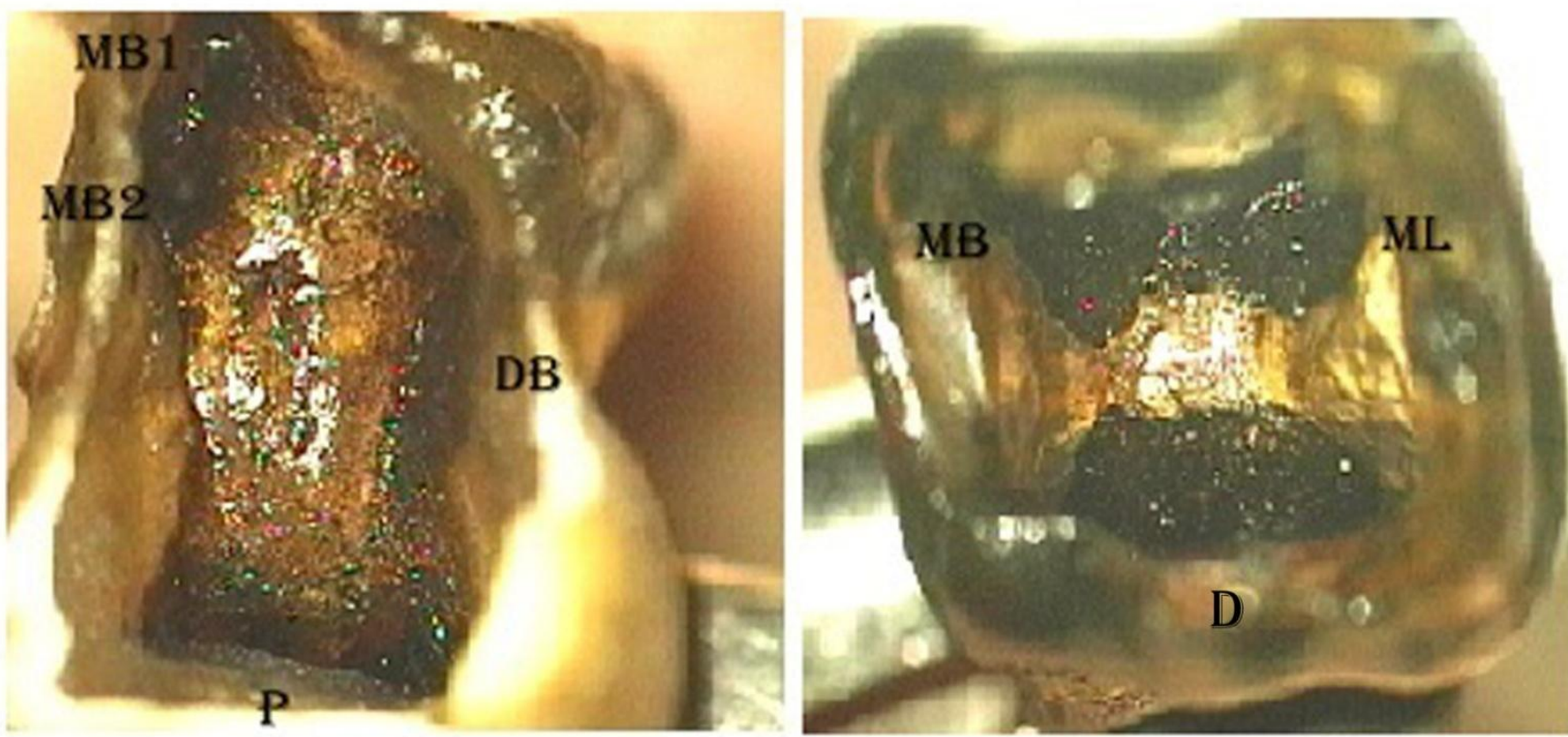

Fig. 2 : maxillary and mandibular molars after clearing techniquz

\section{Statistical analysis}

All the numbers were recorded and statistically analyzed. Analysis was done using Chi square test. 
Table 1 : Showing total orifices detected in each group in combined maxillary and mandibular teeth

\begin{tabular}{|l|c|c|}
\hline Groups & Total number $(\mathbf{n = 4 1 1})$ & Percentage \\
\hline $\begin{array}{l}\text { Group }-1 \\
\text { Naked eye }\end{array}$ & 379 & $92.21 \%$ \\
\hline $\begin{array}{l}\text { Group }-2 \\
\text { Loupes }\end{array}$ & 384 & $93.43 \%$ \\
\hline $\begin{array}{l}\text { Group }-3 \\
\text { Microscope }\end{array}$ & 403 & $98.05 \%$ \\
\hline $\begin{array}{l}\text { Group - } 3 \\
\text { Clearing technique }\end{array}$ & 411 & $100 \%$ \\
\hline
\end{tabular}

Graph 1 : Percentage of total orifices detected among different groups

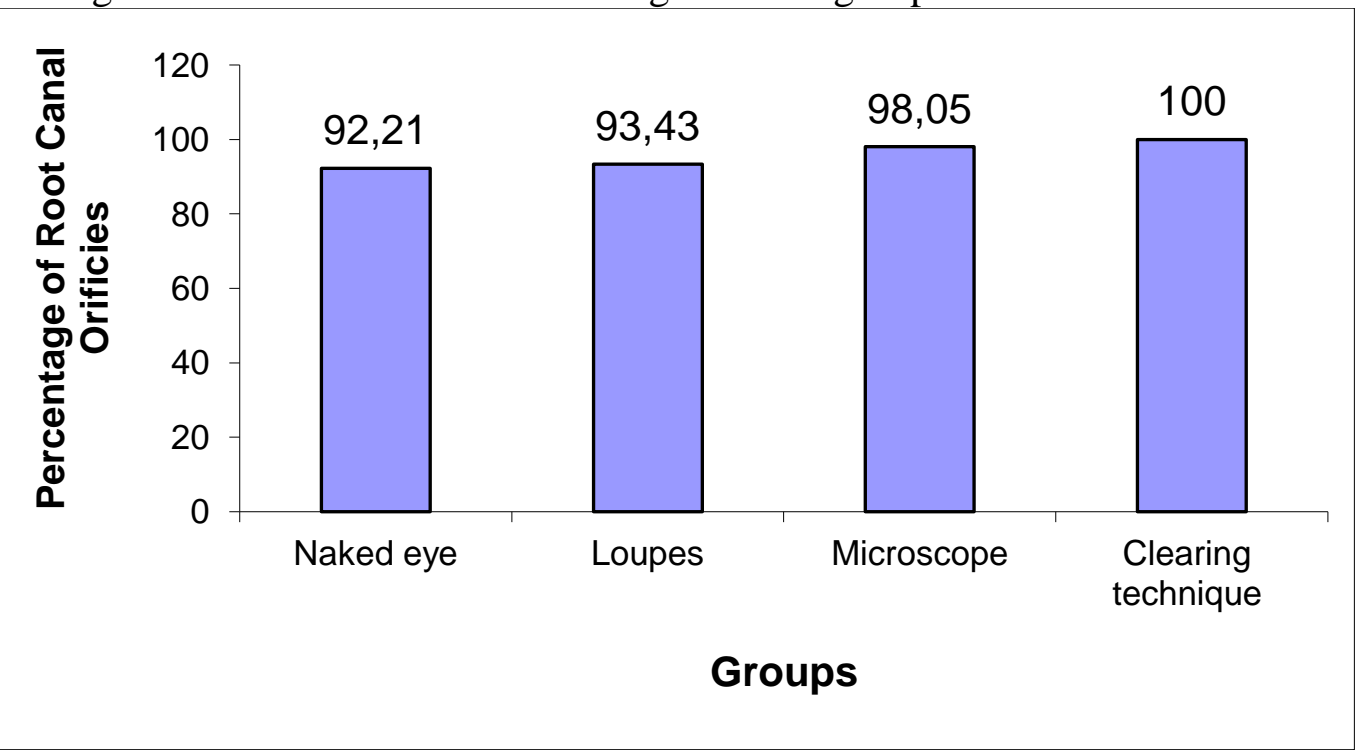

Table 2 : Chi square test applied to table 1

\begin{tabular}{|l|c|c|c|}
\hline Chi square test & $\boldsymbol{\chi}^{\mathbf{2}}$ & $\mathbf{p}$ & Significance \\
\hline Gp 1 vs gp 2 & 0.456 & $\mathrm{p}=4.993$ & $\mathrm{NS}$ \\
\hline Gp 1 vs gp 3 & 15.137 & $\mathrm{p}<0.001$ & $\mathrm{~S}$ \\
\hline Gp 2 vs gp 3 & 10.77 & $\mathrm{p}<0.01$ & $\mathrm{~S}$ \\
\hline
\end{tabular}

\section{Observation and Results}

The results of the study showed that there was significant difference between Groups 1 and 3 and Groups 2 and 3 but there was no significant difference between Groups 1 and 2.

The operating microscope could more accurately detect the orifices than loupes and when no magnification is used.

\section{Discussion}

The main objectives of root canal therapy are thorough shaping and cleaning of all pulp spaces and complete obturation of these spaces with an inert filling material. The presence of an untreated canal may be a reason for failure. A canal may go untreated because the clinician fails to detect it. It is extremely important that the clinicians use all the armamentaria at their disposal to locate and treat the entire root canal system. The complexity of the spaces that must be assessed, shaped, cleaned and filled is remarkable. However even under the most difficult circumstances, current root canal techniques have an exceptionally high rate of success.

The operating microscope is an invaluable tool that aids the endodontist in conventional 
endodontics. The ability to visualize the root canal system in fine detail provides the opportunity to investigate that system more thoroughly and to clean and shape it more efficiently. Today, every challenge existing in the straight portion of the root canal system, even if located in the most apical part, can be easily seen and often solved under the microscope, with magnification and coaxial illumination.

Maxillary molars are widely recognized as being one of the most difficult teeth to treat endodontically. They can present with mild to severe curvatures and can have two or three canals in any root (but most commonly in the mesiobuccal root). Locating the second mesiobuccal canal (MB2) orifice routinely can be difficult as it is often buried under a bridge of dentin. The canal can have a severe curvature to the mesial and the buccal in its coronal section and is usually much smaller than the principal first mesiobuccal canal (MB 1).

The size of access cavity should not be compromised by principles of preserving tooth structure. Although it is important not to remove tooth structure unnecessarily, the use of bonding systems for coronal restorations now means there is less need to preserve tooth structure at all costs. It is beneficial that the access cavity be made larger when the microscope is used. It allows more light to enter the access cavity and thus improves vision. Weller ${ }^{8}$ (1989) et al have stated that there is an increased probability of finding the MB2 canal if the initial access is changed from a classic triangular to a more rhomboidal shape. Straight line access to root canals is important and the opening of the root canals should be in such a position that the instruments can slip down the wall of the access opening and enter the root canal without deviation.It is of fundamental importance to remove all of the roof of the pulp chamber. A dentin bridge may occur due to the secondary dentin formation from aging and/or reparative dentin from carious attack or restorative procedures.
The second mesiobuccal canal (MB2) is reported to occur in more than $90 \%$ of maxillary molars $\left(\right.$ Kulild $\left.^{9}\right)$. On average it is located $1.8 \mathrm{~mm}$ away from the mesiobuccal canal in a palatomesial direction. A protocol involving deepening of the bucco-lingual groove overlying the mesiobuccal root is essential for locating the MB2 orifice $\left(\right.$ Weller, hartwell ${ }^{8}$ ). The groove should not be extended toward the palatal canal but rather in a direction slightly mesial to it, so as to follow the bucco-lingual orientation of the mesiobuccal root. In our study microscope showed the highest number of root canal orifices detected as compared with naked eye and loupes. Khraisat A. ${ }^{10}$ et al in an in vitro study on extracted maxillary molars found a prevalence of MB2 canals to be $77.32 \%$, which is very much in accordance with our study (76.67\%).

Coutinho Filho T. ${ }^{11}$ et al found that with the use of Dental operating microscope, the incidence of MB2 in maxillary first molar increased from 53.7\% to $87.96 \%$ which favours our study. Stropko J J. ${ }^{12}$ found that with experience, scheduled sufficient clinical time, routinely employed dental operating microscope and using specific instruments adapted for microendodontics, MB2 canals can be located in $93 \%$ of maxillary first molars and $60.4 \%$ in second molars

Ahmed $\mathrm{H} \mathrm{A}^{13}$ et al in a study found that in mesial root of mandibular first molars $86 \%$ had two canals, $8 \%$ had one canal, $4 \%$ had three canals and $2 \%$ had other number. In the distal root, $59 \%$ had two canals, $38 \%$ had one canal, $3 \%$ had three canals. This study favours our study except that they had a higher number of two root canals in the distal root and the presence of three canals in the mesial and distal roots. It can be due to the ethnic variation as the teeth studied by them were of Sudanese population. De Carvalho $\mathrm{Mc}^{14}$ et al in a study on mandibular first and second molars found that with the use of operating microscope the number of canals detected increased by $7.8 \%$ than that detected by naked eye. In our study it increased by $7.01 \%$. Overall detection rate of orifices in maxillary and mandibular molar teeth 
by Yoshioka $\mathrm{T}^{15}$ et al was $82.79 \%$ by naked eye, $85.79 \%$ by surgical loupes and $93.17 \%$ by operating microscope. It was less than in our study (92.21\%, 93.43\%, 98.05\% respectively;) because there study was done by less experienced undergraduate students.

Root canal orifices were more easily detected under a microscope than with surgical loupes or naked eye. Magnified views under illumination made it easier to recognize the dentin coverage over the orifice. As a result, the dentin coverage could be more precisely removed. However $1.95 \%$ of all the root canal orifices could not be detected even under a microscope.

\section{Conclusion}

1. The number of orifices detected increased with increasing magnification.

2. Experience of the operator influences the detection rate of root canal orifices.

3. The microscope could more accurately detect orifices statistically than the other two methods.

4. Though the loupes increased the number of detected orifices as compared to naked eye but the difference was not statistically significant.

5. Microscopes may be used in lieu of the loupes owing to its advantages such as higher magnification, more precision, easy documentation.

\section{References}

1. Hess W (1925) Anatomy of the root canals of the teeth of the permanent dentition. Part 1. New York, USA: William Wood and Co, pp. 1- 39

2. Skidmore AE, Bjorndal AM. Root canal morphology of the human mandibular first molar Oral Surg Oral Med Oral Pathol.1971; 32(5):778-84.

3. Pineda F, Kuttler Y. Mesiodistal and buccolingual roentgenographic investiga- tion of 7,275 root canals. Oral Surg Oral Med Oral Pathol 1972; 33(1):101-10.

4. Pineda F. Roentgenographic investigation of the mesiobuccal root of the maxillary first molar. Oral Surg Oral Med Oral Pathol. 1971; 32(5):778-84.

5. Seidberg BH, Altman M, Guttuso J, and Suson M. Frequency of two mesiobuccal root canals in maxillary permanent first molars. J Am Dent Assoc 1973; 87: 852856.

6. Pomeranz $\mathrm{HH}$ and Fishelberg $\mathrm{G}$ The secondary mesiobuccal canal of maxillary molars. J Am Dent Assoc 1974; 88: 119124

7. Vertucci FJ. The significance of mesiobuccal root of the maxillary first molars. US Navy Medicine 1974; 63: 2932.

8. Weller RN, Hartwell GR. The impact of improved access and searching techniques on detection of the mesiolingual canal in maxillary molars. J Endod. 1989 Feb;15(2):82-3. Khraisat A, Smadi L. Canal configuration in the mesio-buccal root of maxillary first molar teeth of a Jordanian population. Aust Endod J. 2007 Apr;33(1):13-7.

9. Kulild JC, Peters DD. Incidence and configuration of canal systems in the mesiobuccal root of maxillary first and second molars. J Endod. 1990 Jul;16(7):311-7

10. Coutinho Filho T, La Cerda RS, Gurgel Filho ED, de Deus GA, Magalhães KM. The influence of the surgical operating microscope in locating the mesiolingual canal orifice: a laboratory analysis. Braz Oral Res. 2006 Jan-Mar;20(1):59-63.

11.Stropko JJ. Canal morphology of maxillary molars: clinical observations of canal configurations. J Endod 1999; 25:446-50. 
12. Ahmed HA, Abu-bakr NH, Yahia NA, Ibrahim YERoot and canal morphology of permanent mandibular molars in a Sudanese population. Int Endod J. 2007 Oct;40(10):766-71.

13.De Carvalho MC, Zuolo ML. Orifice locating with a microscope. J Endod. 2000 Sep;26(9):532-4.

14. Yoshioka T, Kobayashi C, Suda H. Detection rate of root canal orifices with a microscope. J Endod. 02 Jun;28(6):452-3. 